\title{
I fattori che influiscono sul merito creditizio delle aziende agricole: un'applicazione in Campania
}

\author{
Paolo Cupo, Michele Di Domenico*
}

\section{Introduzione}

Il dibattito in corso sull'indirizzo da imprimere alla Politica Agricola Comune (PAC) verso il 2020 contempla, tra le diverse opzioni, anche quella più dirompente imperniata sull'abolizione graduale dei pagamenti diretti per sostituirli con pagamenti limitati per i beni pubblici ambientali e con eventuali pagamenti aggiuntivi per vincoli naturali specifici. Anche nell'ipotesi del mantenimento dello status quo, la necessità di ripartire in maniera più equa $\mathrm{i}$ pagamenti tra un numero più elevato di paesi, lascia intravedere una prospettiva di progressivo ridimensionamento dei contributi pubblici destinati all'agricoltura italiana. Se a ciò si aggiungono le conseguenze negative derivanti dalla crisi economica internazionale e dalla globalizzazione del mercato dei prodotti agro-alimentari, s'impone, soprattutto per gli agricoltori italiani, di vincolare le decisioni aziendali ad un maggior rispetto dei requisiti di competitività ed imprenditorialità. Tali vincoli assumono rilievo più stringente soprattutto per le aziende che producono beni con un basso grado di differenziazione (Peeters, 2009).

Ciò si traduce nella necessità di un aggiustamento strutturale dell'agricoltura italiana, in generale, e campana in particolare, che possa riflettersi positivamente sulla redditività dei fattori di produzione (Blandford, Hill, 2006; Tarditi, 1992). In particolare, tale aggiustamento si esplicita in una crescita degli investimenti in agricoltura, considerando la loro capacità di influenzare

* Università degli Studi di Napoli "Federico II", Centro di Portici. L'articolo è frutto di una rivisitazione e di un aggiornamento della tesi di dottorato di Michele Di Domenico intitolata "Analisi interpretativa del merito creditizio dell'agricoltura campana" finalizzati alla pubblicazione sulla Rivista. 
positivamente la profittabilità e la competitività delle aziende (Huttel, Mubhoff, Odening, 2010), oltre che la capacità di rimborso dei prestiti (Adinolfi e Capitanio, 2009). Le decisioni di investimento, tuttavia, oltre ad essere condizionate dalle opzioni reali, sono fortemente influenzate anche dalle scelte di natura finanziaria - se cade una delle ipotesi sottostanti il costrutto teorico di Modigliani e Miller (1958) di mercati finanziari perfetti - ed in particolare dal livello del credito e dal costo del capitale, se si considera che un'altra fonte "sicura" di approvvigionamento di risorse finanziarie, come i pagamenti diretti, in grado di stimolare gli investimenti anche in assenza di avversione al rischio, molto probabilmente subiranno una contrazione a causa del nuovo corso che verrà impresso alla PAC (Baltas, 1983; Lewis et al., 1988; Dixit, Pindyck, 1994; Vercammen, 2007).

In particolare, il mercato del credito è, più realisticamente, imperfetto per l'esistenza di informazioni asimmetriche tra creditori e debitori generando di conseguenza i fenomeni di adverse selection e moral hazard. L'asimmetria informativa consiste nella sperequata distribuzione delle informazioni tra prestatori e clienti, essendo questi ultimi gli unici in grado di conoscere appieno la propria reale capacità di rimborso e l'effettivo impiego dei fondi presi a prestito con la conseguenza, per i creditori, di sostenere dei costi di transazione per la determinazione del merito creditizio della controparte e per il monitoraggio delle esposizioni (Stiglitz, Weiss, 1981; Benjamin, Phimister, 1997). La selezione avversa si genera, pertanto, a seguito della impossibilità per le banche di entrare in possesso di informazioni inerenti la posizione dei singoli affidati e, quindi, sulla necessità per esse di basarsi su dati medi relativi a gruppi di imprese, finendo con il penalizzare i clienti dotati di una maggiore capacità di rimborso e, viceversa, di premiare quelli ai quali è associabile una più elevata probabilità di insolvenza (Akerlof, 1970). L'azzardo morale, invece, può sorgere per effetto della commistione esistente nell'agricoltura italiana tra impresa e famiglia, per cui i fondi presi in prestito possono essere distolti dalla destinazione per la quale sono stati richiesti, per essere impiegati per soddisfare esigenze di consumo del nucleo familiare (Scaramuzzi, 2002) ${ }^{1}$.

L'acquisizione delle risorse finanziarie necessarie per la realizzazione dei progetti di investimento presentati dalle aziende agricole nell'ambito dei Programmi di Sviluppo Rurale (PSR) delle singole Regioni, anche per integrare i capitali propri o come anticipazione quando il contributo pubblico viene erogato a consuntivo, non può che determinare l'intensificazione dei rapporti tra

1. Va sottolineato, tuttavia, come proprio la presenza di tale commistione tra impresa e famiglia possa produrre un risultato opposto, nel senso di miglioramento della probabilità di rimborso dei prestiti e quindi di riduzione del rischio di credito, a causa dell'esistenza della fonte esterna rappresentata dalla famiglia dell'imprenditore a cui poter attingere risorse per il riequilibrio finanziario (Mauri, 1990). 
istituti di credito ed imprese agricole. Lo studio svolto si inserisce proprio nell'ambito di questo rapporto, soprattutto in considerazione nel nuovo accordo sul capitale Basilea 2, cui devono attenersi gli istituti di credito. Tale accordo acuisce le difficoltà di rapporto tra imprese e banche a seguito delle procedure dettate dai sistemi interni di rating da applicare alla clientela. Difficoltà che sorgono, da un lato, per effetto delle necessarie competenze richieste per valutare la situazione presente e le prospettive future di aziende che nella maggior parte dei casi non hanno l'obbligo di redigere un bilancio (poiché l'accertamento della base imponibile avviene sulla base delle risultanze catastali) - competenze che si sono fortemente ridotte per via della despecializzazione degli istituti di credito determinata dal Testo Unico Bancario (TUB) n. 385/1993 - e dall'altro a seguito delle preoccupazioni che affliggono le imprese agricole che temono ripercussioni negative sulla propria capacità di accesso al credito derivanti dall'applicazione del nuovo accordo e dalla crisi economico-finanziaria internazionale. Preoccupazioni, queste ultime, alimentate oltre che dalla asimmetria informativa tra prestatori e clienti cui si è accennato, che rende oltremodo problematica la valutazione del merito creditizio da parte delle banche, anche da caratteristiche distintive delle imprese che operano in agricoltura e che si riferiscono alla mancanza di documenti contabili prodotti della stragrande maggioranza delle imprese del settore, alle caratteristiche dei cicli produttivi, alla durata degli investimenti (Barry, 2001)2. Infatti, la semplificazione amministrativa vigente in Italia a vantaggio della maggior parte delle imprese agricole organizzate giuridicamente in ditte individuali o società semplici, con il conseguente ridimensionamento della quantità e valenza analitica della documentazione contabile annualmente prodotta, rende difficoltoso l'accertamento della capacità di rimborso e quindi l'attribuzione del rating (Caputo, 2008; Fontana, 2010).

In considerazione di tali difficoltà, lo studio si pone l'obiettivo di valutare il merito creditizio di un campione di aziende agricole rappresentativo dell'agricoltura della regione Campania, soffermandosi in particolare sulle variabili maggiormente significative nell'assegnazione dei rating aziendali, ampliando all'intero settore agricolo regionale l'analisi svolta da Cupo e Di Domenico (2008) riferita ad una specifica azienda floricola campana. Inoltre, la scelta di focalizzare l'analisi nella regione Campania risiede anche nelle caratteristiche della sua agricoltura. Il settore primario regionale, infatti, nel quinquennio 2005-09, ha fatto registrare in base ai dati INEA un valore ag-

2. A tale riguardo, assumono particolare significato due dati che emergono dall'ultimo rapporto Agri2000 riferito al 2010, nel quale si evidenzia come la percentuale del 54\% di un campione di 1.200 aziende agricole abbia finanziato con credito bancario gli investimenti realizzati, contro l' $83 \%$ del 2009, e come si sia assistito nel periodo 2007-10 al progressivo ridursi delle innovazioni introdotte in azienda, passate dal 61 al 29\% (Agri2000, 2010). 
giunto per occupato inferiore dell' $1,2 \%$ alla media nazionale, anche per effetto della patologica polverizzazione fondiaria. Ciò è confermato dalle stime dell'ultima indagine ISTAT sulle strutture e produzioni del 2007, che mostrano un'ampiezza media delle aziende regionali di 3,7 ha/SAU contro i 7,6 della media nazionale (ISTAT, 2009). Tali dati dimostrano come l'agricoltura campana manifesti un fabbisogno di aggiustamento strutturale in scala ancora più accentuata rispetto al dato nazionale, per rispondere più compiutamente alle nuove sfide dettate dai cambiamenti politici ed economici in atto.

I risultati ottenuti dallo studio permetteranno di definire il quadro complessivo della capacità di accesso al credito delle aziende campane e di fornire informazioni utili non solo ai responsabili delle decisioni in merito all'erogazione dei prestiti, ma anche agli stessi imprenditori agricoli che, nella prospettiva di migliorare la loro cultura finanziaria spesso distante dalla logica aziendale esistente nelle aziende operanti in altri settori produttivi (Lucarelli, 2006), dovranno sempre più preoccuparsi di migliorare il profilo delle proprie aziende nella prospettiva di dover richiedere maggiori capitali agli istituti di credito. Infatti, da questo punto di vista, è decisivo conoscere quali sono le variabili maggiormente in grado di incidere sul rating assegnato alle aziende, perché è su di esse che gli imprenditori dovranno intervenire per rafforzare la loro capacità di accesso al credito, così come le banche dovranno essere messe nelle condizioni di poter valutare la probabilità di default di un'azienda dalla conoscenza di alcune variabili chiave di natura esogena ed endogena all'azienda. Tutto ciò nell'ottica, più generale, di migliorare il rapporto tra fabbisogni finanziari delle imprese agricole - che saranno destinati a divenire sempre più articolati e complessi rispetto alla semplice richiesta di fido, anche in considerazione dei maggiori rischi cui sono soggetti gli agricoltori - e la controparte del sistema bancario che, nonostante l'ampliamento di servizi finanziari da collocare presso le imprese agricole previsto dalla riforma del sistema bancario italiano introdotta con il TUB del 1993, non sempre è stata pronta nel cogliere le opportunità legate a tale mutamento della domanda di servizi finanziari da parte dell'imprenditore agricolo (Lucarelli, 2006).

\section{Analisi comparativa della modellistica per la previsione delle insolvenze}

L'esigenza degli istituti di credito di operare una corretta valutazione del merito creditizio dei potenziali clienti, ha spinto molti ricercatori a predisporre dei modelli che fossero in grado di fornire un'accurata stima della probabilità d'insolvenza dei debitori.

La crisi del 1929, con la conseguente esigenza di fornire maggiore stabilità al sistema finanziario, rappresentò il principale incentivo agli studi volti all'individuazione degli indici di bilancio maggiormente espressivi del ri- 
schio d'insolvenza. I lavori di natura esplorativa furono condotti da Fitzpatrick (1932), Smith, Winakor (1935) e Merwin (1942). Il primo, invece, ad utilizzare l'analisi discriminante univariata per la classificazione delle imprese in due gruppi (sane, anomale) fu Beaver (1966; 1968). L'autore prese in esame un campione di 158 imprese (79 sane e 79 insolventi) con dati organizzati in una serie storica di cinque anni e, dall'analisi dei bilanci, calcolò trenta indicatori rappresentativi dell'economicità della gestione, della liquidità e della solidità patrimoniale. Le principali critiche mosse allo studio di Beaver riguardarono l'approccio univariato che, comportando l'analisi degli indici indipendentemente gli uni dagli altri, trascurava completamente l'apporto informativo derivante dall'interazione di più indicatori. Tali critiche segnarono la successiva implementazione di modelli di scoring basati sull'analisi discriminante multivariata (MDA).

La prima applicazione dell'analisi discriminante alle variabili di natura economico-finanziaria dell'impresa si deve ad Altman (1968). L'autore operò su un campione di 66 imprese manifatturiere di cui 33 fallite e 33 sane omogenee per settore, dimensione e periodo d'indagine (1946-65). In particolare, mediante l'applicazione dell'analisi discriminante, Altman costruì un modello con 5 variabili esplicative per classificare le imprese in bankrupt o non-bankrupt, la cui formulazione analitica è di seguito riportata:

$$
Z=0.012 X_{1}+0.014 X_{2}+0.033 X_{3}+0.006 X_{4}+0.999 X_{5}
$$

dove:

$X_{1}=$ Working Capital / Total Assets

$X_{2}=$ Retained earnings / Total Assets;

$X_{3}=$ EBIT / Total Assets;

$X_{4}=$ Market value equity / Book Value of Total Liabilities;

$X_{5}=$ Sales $/$ Total Assets.

Altman identificò anche i valori di cut-off di $\mathrm{Z}$ con cui confrontare il risultato dell'analisi discriminante al fine di assegnare le imprese in esame ad uno dei gruppi definiti a priori. Inoltre, allo stesso autore si deve anche la formulazione del modello denominato EM-Score (derivato dallo Z-Score da cui eredita le variabili esplicative) che permettendo di assegnare, in base allo score della funzione discriminante, l'impresa in esame ad una determinata classe di rating, operò un primo passo verso la predisposizione dei più avanzati sistemi di rating (Altman, 2000). Tale modello è stato di recente applicato alle imprese agricole italiane, nel quale è emerso uno score relativamente migliore per le aziende di medie e grandi dimensioni, rispetto a quelle medio piccole e l'effetto positivo in termini di rating esercitato dagli investimenti (Adinolfi, Capitanio, 2009). 
Nel 1972 Deakin, applicando l'analisi discriminante multivariata allo stesso campione dello studio univariato di Beaver, condusse un confronto tra l'efficacia predittiva dei due metodi. Tale applicazione confermò la valenza dello studio di Beaver anche se alcuni indici, che in precedenza erano stati ritenuti altamente predittivi, risultarono poco significativi sebbene una loro esclusione comportasse una riduzione più che proporzionale della capacità discriminatoria della funzione ${ }^{3}$. La valenza della MDA fu successivamente dimostrata anche da Blum (1974) che disponendo di un campione di 230 aziende (115 fallite e 115 in bonis) e di otto bilanci consecutivi riuscì a condurre un'analisi molto dettagliata ottenendo un modello ad alta affidabilità (percentuale di corretta classificazione del $94 \%$ nell'anno precedente il fallimento).

I primi studi italiani in materia furono condotti da Alberici (1975) su un campione bilanciato di 42 imprese manifatturiere della provincia di Milano. La scelta degli indici, condotta utilizzando il criterio del confronto tra medie, comportò l'individuazione di 7 variabili e la messa a punto di un modello con buona capacità predittiva già dal quinto anno precedente il fallimento. Ancora, si ricorda lo studio di Luerti (1992) che utilizzando un campione non bilanciato di imprese (19 in default e 132 sane) rappresentativo della realtà produttiva italiana, definì una funzione discriminate costituita da 7 variabili esplicative con scores ordinabili in una scala di valori da 1 a 10 .

L'analisi discriminante trovò applicazione anche in numerosi studi inerenti la valutazione del rischio di credito delle imprese agricole. I primi a condurre tali ricerche furono Brinegar e Fettig (1968) i quali dimostrarono come i ritorni attesi netti capitalizzati fossero altamente correlati con le performance del prestito. Successivamente, Beuer e Jordan (1971), Evans (1971), Johnson e Hagen (1973) stimarono funzioni discriminanti con l'intento di collegare le informazioni relative alla concessione dei prestiti con la loro probabilità di successo. Infine, Dunn e Frey (1976), Hardy e Weed (1980) ed Hardy e Adrian (1985) migliorarono l'efficacia dell'analisi discriminante mediante l'utilizzo di un maggior numero di variabili esplicative.

Riassumendo, si può affermare che l'analisi discriminante restituisce al decision maker una classificazione dicotomica delle imprese che, seppure importante, non fornisce però alcuna quantificazione dell' associato rischio di default (Dimitras, Zanakis, Zapounidis, 1996). Partendo da questa considerazione, gli studi si sono incentrati sucessivamente sullo sviluppo di modelli che fossero in grado di dare una misura della probabilità di insolvenza (o fallimento) mediante l'impiego di metodi statistici quali Linear probability models, Logit e Probit.

3. Roggi (2008) sottolinea che i risultati della ricerca di Deakin erano in controtendenza con la precedente visione degli studiosi, convinti che l'eliminazione di un indice poco significativo avrebbe comportato una riduzione dell'efficacia classificatoria al massimo pari al livello di significatività della variabile esclusa. 
Il primo a ricorrere alla Logit Analysis (LA) fu Ohlson (1980) che costruì tre modelli in grado di prevedere i fallimenti d'impresa sui tre anni precedenti il verificarsi dello stesso. Per le applicazioni al settore agricolo si rimanda, invece, ai lavori di Lufburrow, Barry, e Dixon (1984) che utilizzando i dati finanziari di cinque Production Credit Associations (PCA) in Illinois predisposero un Ordinally ranked Limited Dependent Variable probit model (OLDV) che prevedeva l'assegnazione dei debitori ad una delle tre classi di rischio predeterminate (classe 1 a basso rischio, classe 2 a rischio medio e classe 3 ad alto rischio). Gli autori riscontrarono che misure di liquidità, leverage, garanzie, capacità di rimborso e rimborsi storici permettevano di ottenere la migliore capacità classificatoria. Successivamente, Mortensen, Watt, Leistritz (1988) predisposero un Logit Model (LM) per la previsione dei default degli agricoltori del nord Dakota (763 imprese con dati organizzati in uno schema del tipo cross-selection). Di interesse furono anche gli studi di Miller e LaDue (1989) che stimarono due LMs per la previsione delle insolvenze delle aziende zootecniche dell'area di New York. Ancora, Turvey (1991) costruì un modello di credit scoring basato sulla LA per la previsione delle insolvenze dei prenditori della $\mathrm{Ca}$ nada's Farm Credit Corporation utilizzando come variabili predittive del fallimento la liquidità, la profittabilità, leverage, la capacità di rimborso, l'efficienza, il tipo d'impresa e la regione di appartenenza. Tale lavoro risultò estremamente interessante principalmente per il confronto tra LPM, DA, Probit e Logit analysis che indicò come quest'ultimi due metodi restituissero dei risultati solo leggermente migliori rispetto agli altri, confermando quanto già in precedenza indicato da Collins e Green (1981). È interessante notare come studi successivi abbiano dimostrato l'esistenza di variabili trascurate ugualmente utili nella previsione delle insolvenze; in particolare, Gallagher (2001) riportò che le capacità del creditore e il ricorso ad un consulente finanziario potevano essere utilmente impiegate per la previsione del successo di un prestito agrario, mentre Zech e Pederson (2003) indicarono le spese familiari e l'efficienza finanziaria dell'impresa agricola come eccellenti fattori di previsione (Gustafson, Pederson, Gloy, 2005).

Procedendo nella review delle metodologie adottate per la predisposizione dei modelli di credit scoring, vanno ancora indicate l'analisi multicriteria e la programmazione matematica. Tali metodi, pur godendo di un'ampia applicazione nei problemi di analisi discriminante, non trovarono un altrettanto considerevole utilizzo nella previsione dei fallimenti delle imprese agricole. È comunque interessante citare il lavoro di Ziari, Leatham, Turvey (1995) che dimostrarono come l'applicazione della programmazione matematica in un modello di credit scoring per i prestiti al settore agricolo, restituisse dei risultati altrettanto buoni di quelli ottenibili con l'impiego degli altri metodi statistici (Logit, Fisher's linear discriminant function).

Tra le metodologie alla base dei moderni modelli di scoring (e di rating), uno spazio significativo è occupato da tutta quella serie di metodi riconduci- 
bili alla disciplina dell'Intelligenza Artificiale. In generale, l'obiettivo di tale disciplina è di realizzare una macchina in grado di risolvere "qualunque" problema in quanto non settata su un determinato algoritmo, ma in grado di creare essa stessa un algoritmo a partire dal problema osservato.

Nell'ambito delle tecniche adottate per la previsione dei fallimenti d'impresa facenti capo alle reti neurali, la più utilizzata è la Back Propagation Neural Network (BPNN). Ravi Kumar P., Ravi V. (2007), conducendo un'ampia analisi della letteratura relativa alle metodologie per la previsione dei fallimenti d'impresa, notò che in ogni ricerca in cui fosse stato condotto un confronto tra le varie tecniche statistiche (DA, Logit, Probit, ecc.) e quelle di Neural Network (BPNN in particolare), si giungeva alla medesima conclusione di migliore performance di quest'ultime.

Ai fini della nostra review è interessante menzionare un'ulteriore tecnica che negli ultimi anni ha iniziato a trovare applicazione nel campo finanziario ed in particolare nella predisposizione di alcuni modelli di rating. Ci si riferisce alla Support Vector Machines (SVM): un insieme di metodi di supervised learning 4 usati a fini di classificazione o regressione. Riprendendo le conclusioni del lavoro di Ravi Kumar P., Ravi V. (2007), richiamato in precedenza, è possibile ottenere delle prime indicazioni sulla capacità operativa della SVM nella risoluzione dei problema di "banckrupt" (o di insolvenza nei modelli di rating) risultando questi ultimi, per tutti gli esempi esaminati, più performanti delle classiche tecniche statistiche ed anche della BPNN.

\section{Valutazione del merito creditizio di un campione rappresentativo di aziende agricole della regione Campania}

\subsection{Il campione di riferimento}

Ai fini dell'analisi, sono stati presi in esame i dati della Rete di Informazione Contabile in Agricoltura (RICA) relativi agli anni 2005, 2006 e 2007 per un campione di 368 aziende agricole campane.

La scelta di impiegare i dati RICA trova giustificazione, oltre che nell'ufficialità degli stessi, anche nella mole di variabili economiche e non, rilevate dalla rete, che si ritiene possano correttamente fungere da base informativa per il modello di rating che si prevede di impiegare. Inoltre, l'esigenza di disporre per la determinazione del rating di almeno due bilanci a consuntivo per ciascuna azienda, ha reso necessaria l'estrazione dal campione 2005-07

4. È una tecnica di machine learning (disciplina che disegna e sviluppa algoritmi che permettono ai computer di "imparare" dai dati) usata per dedurre una funzione da un training data. 
delle sole aziende presenti in ciascuno dei tre esercizi rilevati, ottenendo in tal modo un campione costante sull'orizzonte temporale di riferimento. $\mathrm{E}$ interessante notare, che l'estrazione dal campione RICA delle sole aziende rilevate in tutti gli anni considerati, ha restituito per il 2007 un campione statisticamente non dissimile da quello complessivo. A tale scopo, per le variabili di stratificazione del campione, è stato calcolato l'indice di dissomiglianza $\left(\mathrm{ID}_{\mathrm{s}}\right)$ (Guarini, Tassinari, 1990):

$$
I D s=\frac{1}{2} \sum h\left|\frac{A_{i h}}{A_{i 0}}-\frac{A_{0 h}}{A_{00}}\right|
$$

dove:

$\mathrm{A}_{\text {ih }}=$ indica il numero di aziende dell'h-mo Ordinamento Tecnico Economico (OTE), Unità di Dimensione Economica (UDE), zona altimetrica presenti nel campione costante;

$\mathrm{A}_{\mathrm{i} 0}=$ indica il numero complessivo di aziende del campione costante;

$\mathrm{A}_{0 \mathrm{~h}}=$ indica il numero di aziende dell' $h$-mo OTE (UDE, zona altimetrica) presenti nel campione RICA 2007;

$\mathrm{A}_{00}=$ indica il numero complessivo di aziende del campione RICA 2007.

L'indice assume valore zero se il campione costante presenta una distribuzione di aziende, rispetto alla variabile in esame, identica a quella del campione complessivo e valore uno quando tutte le aziende del campione costante sono concentrate in una singola modalità dell' $h-m a$ variabile, a differenza di quanto si verifica nel campione RICA 2007. I risultati mostrano per le variabili di stratificazione valori dell' $\mathrm{ID}_{\mathrm{s}}$ pressoché prossimi allo zero, a dimostrazione della sostanziale "somiglianza" dei due campioni a confronto $\left(\mathrm{ID}_{\mathrm{s} \_} \mathrm{OTE}=0.065 ; \mathrm{ID}_{\mathrm{s} \_} \mathrm{UDE}=0.047 ; \mathrm{ID}_{\mathrm{s} \_}\right.$Altimetria $\left.=0.063\right)$.

\subsection{Mappa dei rating delle aziende del campione}

L'obiettivo della ricerca è di individuare le variabili maggiormente esplicative del merito creditizio delle imprese agricole non soggette all'obbligo di redazione dei bilanci. A tale scopo è fondamentale conoscere il rating di ciascuna azienda del campione in esame, in modo da adottarlo come variabile dipendente per il successivo modello di regressione. Tali rating sono stati assegnati dall'Istituto di Servizi per il Mercato Agricolo e Alimentare (ISMEA) ${ }^{5}$ che ha

5. ISMEA è l'ente economico di diritto pubblico che opera per conto del Ministero delle Politiche Agricole Alimentati e Forestali (MIPAAF) nell'ambito dei finanziamenti agevolati e 
applicato il modello denominato "SME - Small and Medium Enterprises Model" specifico per le imprese agricole prive di bilanci. In termini generali, il modello SME può essere collocato nella classe dei modelli di rating fondati sulla Probit Analysis e classificato tra i "Non-structural models"6. In particolare, il modello è basato su un approccio "data-driven" e "foundamental analysis" (o expert-driven) in cui la componente esperta deriva dalla fusione dell'esperienza di Moody's nella modellistica dei rating con le conoscenze di ISMEA del settore agricolo nazionale. L'analisi probit ha permesso a Moody's di identificare in termini multivariati le variabili maggiormente significative nella predizione dei default e di implementare un software, RiskAnalyst ${ }^{\mathrm{TM}}$ 3.0, specifico per la determinazione del rating delle aziende agricole non soggette all'obbligo di bilancio. Il programma è costruito seguendo la metodologia delle SCORECARD (schede di valutazione a punteggio) attraverso le quali tutte le informazioni quantitative e qualitative relative alle aziende oggetto di studio sono sintetizzate, combinate e pesate. Attualmente il software si compone di tre scorecard una per ciascuno dei seguenti fattori di rischio:

1. mercato/industria;

2. business;

3. risultati economico-finanziari.

Partendo dai dati quanti/qualitativi riportati nelle singole schede a punteggio ed attraverso l'applicazione di pesi prestabiliti, il software (modello $\mathrm{SME}$ ) è in grado di assegnare un giudizio di rating alle aziende in esame.

$\mathrm{Si}$ evidenzia che la scelta di ricorrere ad ISMEA per l'assegnazione dei $\mathrm{ra}$ ting, è stata condotta tenendo in considerazione non solo il ruolo istituzionale rivestito dall'Istituto, ma anche i risultati delle indagini condotte presso i principali istituti di credito operanti a livello nazionale nel settore primario, che hanno evidenziato l'assenza (in alcuni casi l'esplicita volontà di utilizzare i modelli ISMEA) o l'impossibilità ad uso esterno dei modelli interni di valutazione.

In linea con i risultati attesi da ISMEA, le aziende del campione costante RICA si ripartiscono sulla scala dei rating secondo una funzione di distribuzione Normale, che evidenzia come solo una piccola parte del campione ricada in una classe di rating particolarmente bassa (Graf. 1).

garanzie alle imprese agricole italiane. In particolare, ISMEA in collaborazione con la società di rating Moddy's KMV ha predisposto dei modelli di rating specifici per le imprese agricole, che attualmente rappresentano il principale punto di riferimento per gli operatori del settore.

6. Si definiscono "Non structural" i modelli la cui specificazione non è fondata sulla teoria economica. 
Graf. 1 - Mappatura dei rating delle aziende agricole del campione costante RICA 2005/07

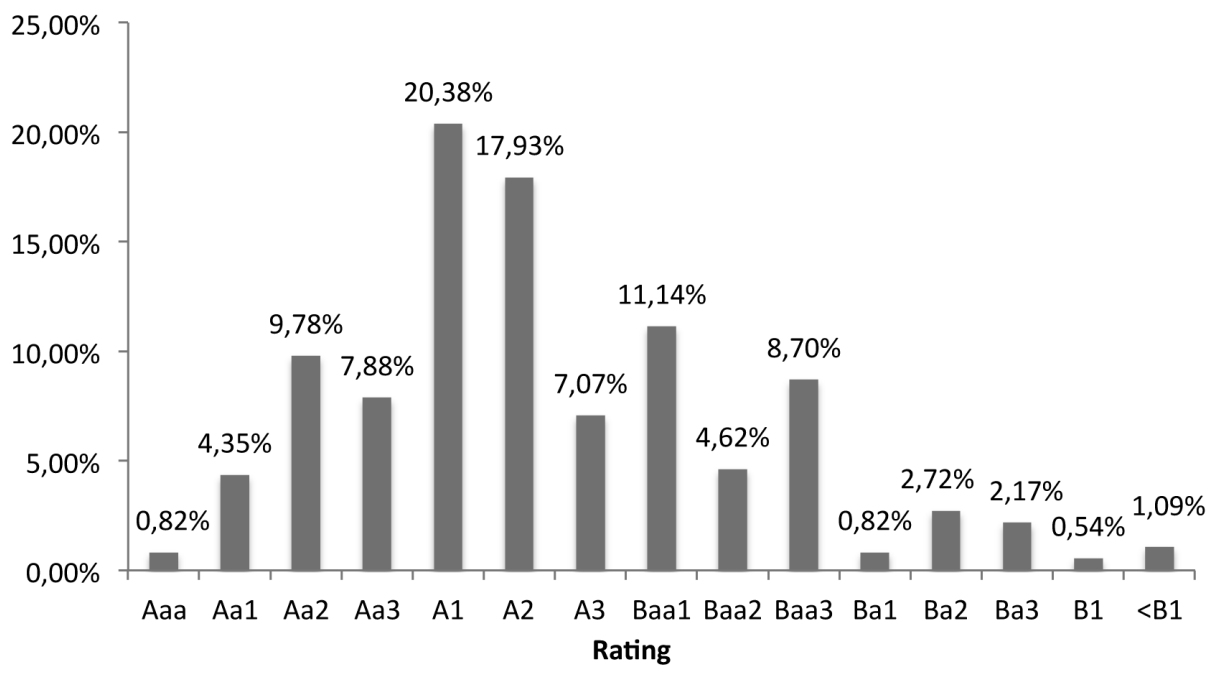

Ciò appare ancora più evidente se si opera un raggruppamento delle categorie di rating per livello di solvibilità ${ }^{2}$. Infatti, circa il $38 \%$ delle aziende rientra nella classe "solvibile" con un'alta percentuale $(22,83 \%)$ collocata nella categoria "altamente solvibile". Nettamente minore la percentuale di aziende "rischiose" $(3,8 \%)$ con il restante $35 \%$ circa di aziende nella classe di vulnerabilità.

Tali risultati sembrerebbero indicare una condizione di buona capacità di accesso al credito per le aziende agricole in esame.

Tuttavia, dall'analisi degli indici statistici descrittivi delle singole aziende del campione, non è stato possibile identificare una chiave interpretativa dei risultati conseguiti in termini di rating. L'esigenza di migliorare la portata esplicativa degli scores attribuiti alle aziende, ha imposto di procedere alla individuazione delle variabili indipendenti del merito creditizio, impiegando un modello che fosse in grado di replicare i risultati assegnati da ISMEA.

7. Le classi sono così ottenute: "altamente solvibile" da "Aaa" a "Aa3"; "solvibile" da "A1" a "A2"; "vulnerabile" da "A3" a "Ba2"; "rischiosa" inferiore o uguale a "Ba3". La classificazione è stata ottenuta utilizzando come riferimento una tabella di codifica dell'ISMEA, che assegna a determinati intervalli di probabilità di default le corrispondenti classi di solvibilità. 


\section{Individuazione delle variabili esplicative del merito creditizio}

\subsection{Il modello statistico utilizzato}

Essendo in presenza di variabili dipendenti multinomiali categoriche e ordinali (classi di solvibilità) si è deciso di utilizzare un modello del tipo Ordered Probit che restituisce stime maggiormente efficienti rispetto ai modelli che trascurano l'informazione aggiuntiva del ranking dalla variabile dipendente (Winkelmann, Boes, 2006).

Il modello Oprobit, in generale, è caratterizzato da un processo continuo e non osservabile:

$$
y_{i}^{*}=x_{i}^{\prime} \beta+u_{i}(i=1, \ldots, n)
$$

costituito da una componente deterministica $x^{\prime}{ }_{i} \beta$ e una random $u_{i}$ che si assume essere indipendente e identicamente distribuita con funzione di distribuzione normale standardizzata $(u)$ a media zero e varianza costante. Ovviamente è possibile osservare solo $y_{i}$ (le classi di solvibilità con valori $\left.1, \ldots, J\right)$ e pertanto è necessario individuare un meccanismo che permetta di collegare $y_{i}^{*}$ a $y_{i}$. Tale meccanismo prende il nome di "Threshold mechanism" e nel nostro caso assume che:

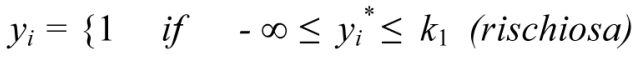

$$
\begin{aligned}
& 2 \text { if } k_{1}<y_{i}^{*} \leq k_{2} \text { (vulnerabile) } \\
& 3 \text { if } k_{2}<y_{i}{ }^{*} \leq k_{3} \text { (solvibile) } \\
& 4 \text { if } k_{3}<y_{i}^{*} \leq+\infty \text { (altamente solvibile) }
\end{aligned}
$$

dove: $k$ sono i $J$-1 parametri soglia incogniti.

La probabilità che $y_{i}$ assuma un certo valore sarà:

$$
\pi_{i j}=P\left(y_{i}=j \mid x_{i}\right)=\Phi\left(k_{j}-x_{i}^{\prime} \beta\right)-\Phi\left(k_{j-1}-x_{i}^{\prime} \beta\right) \quad j=1, \ldots \ldots, J
$$

Si precisa che nel modello Oprobit il valore del coefficiente stimato non indica la variazione nella probabilità di un dato risultato dovuta alla variazione unitaria della variabile esplicativa. Inoltre, solo l'impatto sulla probabilità stimata di appartenenza al top rating e al bottom rating - dovuto alla variazione in una variabile esplicativa - è univoco. Ad esempio, nel nostro caso, se $\beta$ è positivo di certo un incremento dell'associata variabile $x$ aumenta la probabilità di avere "altamente solvibile" e riduce la probabilità di avere "ri- 
schiosa". Tuttavia l'effetto sulle probabilità stimate per le classi intermedie è ambiguo (Greene, 2000).

\subsection{Applicazione del modello al campione di aziende}

Il nostro obiettivo è di realizzare un modello con la più elevata capacità di corretta classificazione delle aziende in termini di solvibilità. A tale scopo alcuni possibili regressori sono stati individuati nella banca dati RICA sfruttando anche le indicazioni provenienti dalle evidenze statistiche dei modelli per la previsione delle insolvenze presentati in precedenza (Tab. 1). La variabile dipendente (score) è stata ottenuta accorpando le classi di rating del modello ISMEA in 4 categorie con livello di solvibilità crescente (1 "rischiosa" 2 "vulnerabile" 3 "solvibile" 4 "altamente solvibile") in risposta all'esigenza di disporre per l'analisi di una variabile dipendente con un contenuto numero di possibili alternative.

Il modello che ha determinato la migliore classificazione delle aziende è riportato nella tabella 2 . Si sottolinea che per la scelta del modello sono state seguite le indicazioni provenienti dai criteri informativi AIC (Akaike Information Criterion) e BIC (Bayesian Information Criterion). Tali criteri si basano sull'intuizione che esista una tensione tra il "fit" del modello, che potrebbe essere migliorato aumentando la complessità dello strumento, e il principio di parsimonia che favorisce i modelli più semplici. Tale tensione implica che l'inclusione di ulteriori parametri è giustificata solo se il risultante miglioramento in termini di "fit" compensa in modo sufficiente la perdita di parsimonia. In generale, il modello con più bassi criteri informativi è preferito (Cameron, Trivedi, 2005). Il modello riportato nella tabella 2, pertanto, rappresenta il miglior modello stimato con valore più basso degli "information criterion" oltre che un miglior "fit" come espresso dall'incremento del Log pseudolikelihood, in relazione anche ad una più elevata capacità di corretta classificazione delle aziende.

Per la determinazione della capacità di corretta classificazione del modello sono state calcolate le predicted probabilities ed è stata costruita la tabella di confronto tra le classi di solvibilità osservate (fonte ISMEA) e quelle predette (Tab. 3). In particolare, un'azienda è assegnata ad una certa classe di solvibilità se la probabilità predetta di appartenenza a quella data classe è la massima fra tutte le probabilità stimate:

$$
P\left(y_{i}=j\right)=\max \hat{P}\left(y_{i}=j\right) \quad j=1, \ldots . . J
$$

La diagonale evidenzia il numero di aziende per le quali la classe osservata e predetta coincidono e restituisce, di conseguenza, il grado di corretta 
Tab. 1 - Lista delle principali variabili esaminate

\begin{tabular}{|c|c|c|}
\hline VARIABILE & CODICE & DESCRIZIONE \\
\hline Superficie & $\overline{\mathrm{SAU}}$ & Misura la dimensione fisica \\
\hline Dimensione economica & UDE & Misura la dimensione economica \\
\hline Redditività della terra & RLAND & $\mathrm{PLV} / \mathrm{SAU}$ \\
\hline Redditività del lavoro & RJOB & PLV/ UL totali \\
\hline Return On Equity & ROE & Misura la redditività del capitale proprio \\
\hline Return On Investment & ROI & Misura la redditività del capitale investito \\
\hline Return On Sales & ROS & Misura la redditività delle vendite \\
\hline Valore Aggiunto Netto & VAN & Misura la variazione del VAN \\
\hline Intesità dei premi & PRZ & Misura l'incidenza dei premi sul reddito netto \\
\hline Dipendenza da capitale & CAPDEP & $\begin{array}{c}\text { Dummy che assume valore } 1 \text { per le aziende } \\
\text { ad alta dipendenza e valore } 0 \text { in caso } \\
\text { contrario }\end{array}$ \\
\hline Leverage & LEV & $\begin{array}{l}\text { Misura il grado di dipendenza da capitali } \\
\text { esterni }\end{array}$ \\
\hline Intensità aziendale riferita alla terra & GIAT & $\begin{array}{c}\text { (Investimenti fondiari }+ \text { Capitale d'esercizio/ } \\
\text { SAU) }\end{array}$ \\
\hline Intensità aziendale riferita al lavoro & GIAL & $\begin{array}{c}\text { (Investimenti fondiari + Capitale d'esercizio/ } \\
\text { UL totali) }\end{array}$ \\
\hline Intensità d'esercizio riferita al lavoro & GIEL & $\begin{array}{c}\text { (Capitale d'esercizio/UL totali) misura la } \\
\text { variazione del grado di intensità d'esercizio } \\
\text { del lavoro. }\end{array}$ \\
\hline Intensità d'esercizio riferita terra & GIET & $\begin{array}{c}\text { (Capitale d'esercizio/SAU) misura la } \\
\text { variazione del grado di intensità d'esercizio } \\
\text { del fattore terra. }\end{array}$ \\
\hline Intensità fondiaria riferita alla terra & GIFT & $\begin{array}{l}\text { (Investimenti fondiari SAU) misura la } \\
\text { variazione del grado di intensità fondiaria del } \\
\text { capitale terra. }\end{array}$ \\
\hline Intensità fondiaria riferita al lavoro & GIFL & $\begin{array}{l}\text { (Investimenti fondiari/ UL totali) misura la } \\
\text { variazione del grado di intensità fondiaria del } \\
\text { lavoro. }\end{array}$ \\
\hline OTE & $\begin{array}{c}\text { ALLEVAMENTI } \\
\text { OTICOLTURA } \\
\text { FLORICOLTURA } \\
\text { ARBORICOLTURA } \\
\text { OLIVICOLTURA } \\
\text { SEMINATIVI }\end{array}$ & $\begin{array}{l}\text { Dummy che assume valore } 1 \text { per le aziende } \\
\text { dell'ordinamento indicato e } 0 \text { per le aziende } \\
\text { appartenenti ad un altro OTE. }\end{array}$ \\
\hline Management & FAMFARM & $\begin{array}{l}\text { Dummy che assume valore } 1 \text { per la forma di } \\
\text { conduzione diretta e valore } 0 \text { per le altre } \\
\text { forme di conduzione. }\end{array}$ \\
\hline Vendita prodotti & DSALES & $\begin{array}{l}\text { Dummy che assume valore } 1 \text { in presenza di } \\
\text { vendita diretta e } 0 \text { in caso contrario. }\end{array}$ \\
\hline Qualità & DQLT & $\begin{array}{l}\text { Dummy che assume valore } 1 \text { per aziende che } \\
\text { realizzano produzioni certificate e } 0 \text { in caso } \\
\text { contrario. }\end{array}$ \\
\hline
\end{tabular}


Tab. 2 - Ordered Probit Regression (la variabile dipendente è score)

\begin{tabular}{lcc}
\hline \multicolumn{1}{c}{ Indipendent variables } & Coeff. & Robust Std. Err. \\
\hline CAPDEP & $-1.2693^{* * *}$ & .2527 \\
NAV & $.0179^{* * *}$ & .0026 \\
ROI & $.0016^{* *}$ & .0007 \\
FAMFARM & $.5296^{* * *}$ & .1778 \\
FLORICULTURE & $1.3380^{* * *}$ & .3190 \\
HORTICULTURE & $.2845^{*}$ & .1558 \\
PERMANENTCROP & -.1251 & .1822 \\
OLIVES & $1.4403^{* * *}$ & .2647 \\
FIELDCROP & $1.3322^{* * *}$ & .3170 \\
GIET & $.0094^{* * *}$ & .0033 \\
GIFT & $-.0012^{* * *}$ & .0004 \\
DSALES & $.5012^{* *}$ & .2233 \\
\hline \hline /cut1 & -1.8821 & .2346 \\
/cut2 & .2575 & .1911 \\
/cut3 & 1.9380 & .2022 \\
\hline \hline Number of obs: & 368 & \\
Wald chi2(16): & 162.24 & \\
Prob $>$ chi2: & 0.000 & \\
Pseudo R ${ }^{2}:$ & 0.3030 & \\
Log pseudolikelihood: & -306.9439 & \\
\hline
\end{tabular}

Nota: come correzione dall'eteroschedasticità il modello utilizza robust standard errors (White, 1980). $* * * \mathrm{p}<0.01 ; * * \mathrm{p}<0.05 ; * \mathrm{p}<0.1$.

Tab. 3 - Classificazioni per il modello Oprobit con quattro classi di solvibilità

\begin{tabular}{|c|c|c|c|c|c|}
\cline { 2 - 5 } \multicolumn{1}{c|}{} & \multicolumn{5}{c|}{ Classi osservate } \\
\hline Classi predette & Rischiosa & Vulnerabile & Solvibile & Alta solvibilità & Totale \\
\hline Rischiosa & 2 & 0 & 0 & 0 & 2 \\
\hline Vulnerabile & 12 & 95 & 33 & 2 & 142 \\
\hline Solvibile & 0 & 34 & 88 & 33 & 155 \\
\hline Alta solvibilità & 0 & 0 & 20 & 49 & 69 \\
\hline Totale & 14 & 129 & 141 & 84 & 368 \\
\hline
\end{tabular}

Corretta classificazione: $63,32 \%$

classificazione. Tutto ciò che si trova al di sotto o al di sopra della diagonale rappresenta un errore.

In particolare, nel nostro modello gli errori di sovrastima e sottostima del giudizio sono rispettivamente del $23,24 \%$ e del $18,64 \%$, mentre la percentua- 
le di corretta classificazione è del $63,32 \%$. Tale percentuale è ritenuta soddisfacente se si considera l'impossibilità di risalire in modo completo (variabili non incluse nella RICA) e preciso al set di variabili esplicative adottate dal modello ISMEA. Inoltre, il tasso di corretta classificazione ottenuto è relativamente alto soprattutto se confrontato con le performance dei modelli di scoring per le imprese agricole presenti in letteratura che utilizzano un numero inferiore di classi (ad esempio: 85\% per Bauer e Jordan, $62 \%$ per Johnson e Hagan, $75 \%$ per Dunny e Frey, $81 \%$ per Hardy e Weed, $66 \%$ per Lufburrow, Barry e Dixon ${ }^{9}, 88 \%$ per Mortensen, Watt e Leistritz, $73 \%$ per Miller e La Due, 69\% per Turvey). A conferma di ciò, il modello stimato raggiungere risultati ottimi accorpando le aziende delle classi 1 e 2 nella categoria "scarsa solvibilità" e le aziende delle classi 3 e 4 nella categoria "buona solvibilità" (tasso di corretta classificazione dell' $81,52 \%$ ). In tal modo si opera una classificazione in solo due classi sfruttando però al contempo, mediante il modello Ordered (stima condotta su quattro classi), l'informazione proveniente dal ranking della variabile dipendente. La tabella 4 riporta il confronto tra le classi osservate e quelle predette dal modello utilizzando due livelli di solvibilità.

Tab. 4 - Tabella di confronto con due classi di solvibilità

\begin{tabular}{|c|c|c|c|}
\cline { 2 - 3 } \multicolumn{1}{c|}{} & \multicolumn{2}{c|}{ Classi osservate } & \multirow{2}{*}{} \\
\hline Classi predette & scarsa solvibilità & buona solvibilità & Totale \\
\hline scarsa solvibilità & 107 & 32 & 228 \\
\hline buona solvibilità & 36 & 193 & 140 \\
\hline Totale & 225 & 143 & $\mathbf{3 6 8}$ \\
\hline
\end{tabular}

Corretta classificazione: $81,52 \%$

\subsubsection{Validazione del modello}

È stata condotta una validazione out-of-sample in modo da determinare la capacità di corretta classificazione del modello quando applicato ad aziende non incluse nel sample. In termini operativi, si è provveduto ad estrarre dal

8. L'accuratezza di previsione del modello può essere determinata anche confrontando lo score di ciascuna azienda con i valori soglia. Con questo metodo le performance del modello migliorano leggermente con una percentuale di corretta classificazione del 64,4\%.

9. Tale risultato era ottenuto confrontando le probabilità predette dal modello con tre classi di rischio e la classificazione dei Production Credit Associations (PCA). 
campione RICA, in modo casuale e senza ripetizione, il 95\% di aziende del campione originario. Sul nuovo sample, costituito dal 95\% di aziende estratto, è stato "lanciato" il modello Oprobit e su quelle escluse (5\%) condotta la previsione e calcolata la percentuale di corretta classificazione (out-of-sample forecasting). Poiché i risultati della previsione dipendono, ovviamente, dalle caratteristiche del $5 \%$ di aziende escluse, è stata condotta una simulazione Montecarlo con estrazione casuale ripetuta 1.000 volte del $95 \%$ di aziende dal campione costante RICA. In termini medi, la percentuale di corretta classificazione ottenuta dal modello quando applicato ad aziende fuori dal sample è del $61 \%$. Tuttavia, operando su una classificazione in due classi di solvibilità si raggiunge un valore medio di corretta previsione dell' $80,6 \%$.

\subsection{I risultati}

Il modello evidenzia la significatività per le variabili economiche quali la dipendenza da capitale - calcolata come rapporto delle spese per il rinnovo/ammodernamento degli investimenti in capitale fondiario e agrario sul Margine Operativo Lordo (MOL) - e la variazione del Valore Aggiunto Netto. In particolare, il segno negativo per CAPDEP indica che all'aumentare dell'impatto delle spese per il rinnovo e l'ammodernamento dei capitali sulle performance aziendali, si riduce la probabilità per le aziende di migrare da una classe di solvibilità peggiore ad una migliore; mentre il segno positivo di VAN denota un andamento opposto derivante dalla maggiore disponibilità monetaria che viene a realizzarsi nel confronto tra i due esercizi. Significativo anche il coefficiente stimato per l'indice ROI, il cui segno positivo mostra che le aziende in grado di generare una maggiore redditività operativa dal capitale investito hanno una più alta probabilità di essere associate ad un buono score ${ }^{10}$.

Molto interessanti sono i risultati dell'oprobit anche per alcune variabili di natura qualitativa come la presenza di filiera corta DSALES e la forma di conduzione FAMFARM ${ }^{11}$. Si nota, infatti, che le aziende che aderiscono alla filiera corta, potendo probabilmente spuntare dalla vendita diretta dei prodotti aziendali prezzi più alti rispetto alla cessione degli stessi all'ingrosso, sono considerate maggiormente solvibili. Inoltre, la significatività e il coefficiente

10. Dalla regressione è stato escluso l'indicatore ROE per collinearità con la variabile ROI derivante principalmente dal ridotto o assente ricorso a fonti esterne di finanziamento per le aziende del campione in esame.

11. Più in generale, Crivellaro (2008) ritiene che, tra i fattori di rischio, le variabili di natura qualitativa (serietà e capacità dell'imprenditore e del management, livello tecnologico dell'impresa, ecc.) incidono sullo score finale per non più del $10-15 \%$. 
positivo per la variabile FAMFARM indicano che le aziende condotte in economia diretta hanno una maggiore probabilità di ottenere una classificazione migliore di quelle condotte in altre forme (capitalistica, mezzadria, ecc.). Tale risultato trova probabilmente il suo fondamento non tanto nelle performance economiche, quanto nella maggiore volontà/capacità implicita delle aziende a conduzione diretta di far fronte agli impegni finanziari assunti e derivante dalla forte commistione impresa-famiglia.

Coefficiente positivo anche per GIET che esprime il grado di intensità d'esercizio del fattore terra. Un incremento della dotazione d'esercizio (macchine, attrezzature, bestiame, ecc.) per unità di superficie implica, quindi, un aumento della probabilità di appartenenza ad una classe più alta di solvibilità. È invece negativo il coefficiente della variabile riferita al grado di intensità fondiaria del fattore terra GIFT, a dimostrazione che un ulteriore incremento degli investimenti fondiari per unità di superficie conduce ad un peggioramento della probabilità di ottenere un buon giudizio di merito creditizio. In effetti, l'impatto negativo della variabile GIFT trova spiegazione nel fatto che un aumento della dotazione fondiaria per ettaro comporta, di solito, un'elevata immobilizzazione di capitale con difficoltà per l'azienda di generare in tempi brevi liquidità, mediante alienazione dei beni, per far fronte agli impegni assunti con la banca; inoltre un incremento del capitale fondiario genera un aumento dei costi fissi legati al mantenimento dei cespiti con impatto negativo sulle performance aziendali. Va anche sottolineato che, nel rispetto della definizione di merito di credito, non si tiene conto della possibilità degli investimenti fondiari di essere utilizzati come strumenti di garanzia a supporto della richiesta di affidamento che come tali agiscono solo post rating.

Passando ad esaminare le variabili di settore si nota che le aziende dell'OTE ARBORICOLTURA non manifestano differenze statisticamente significative rispetto alla media delle aziende appartenenti all'ordinamento ZOOTECNIA (dummy esclusa dalla regressione); viceversa quelle con ordinamento FLORICOLTURA, ORTICOLTURA (significatività entro il 10\%), OLIVICOLTURA e SEMINATIVI hanno una maggiore probabilità di appartenere a classi di solvibilità migliori. Tale risultato va ovviamente letto in termini di capacità della singola impresa di restituire nei tempi stabiliti un prestito e non certo di performance economiche in senso stretto dello specifico ordinamento produttivo. Infatti, facendo anche riferimento all'esperienza di ISMEA, si evidenzia che le aziende olivicole e seminative spesso tendono a non indebitarsi oppure si indebitano in modo più ponderato rispetto ad altre realtà aziendali più performanti manifestando minori default. Tale spiegazione sembrerebbe trovare supporto anche nelle dinamiche delle sofferenze analizzate dall'INEA (2009), che evidenzia una minore concentrazione di tali eventi negativi proprio nei piccoli affidatari. 


\section{Conclusioni}

Lo studio svolto ha cercato di analizzare il problema dell'individuazione delle variabili in grado di influenzare l'offerta di credito per le imprese agricole campane, alla luce dell'applicazione dei sistemi di rating introdotti dall'accordo Basilea 2, sostanzialmente confermati anche nella nuova versione di Basilea 3. Problema, questo, di grande attualità se si considera come la disponibilità di fondi si rifletta sulla capacità di investimento del settore e quindi sulla potenzialità di crescita dell'agricoltura regionale.

Sul piano della capacità di accesso al credito, dalla classificazione nelle quattro classi di rating individuate emerge un risultato abbastanza favorevole per le aziende agricole regionali, se si considera che circa il $60 \%$ di esse si dimostra in grado di rispettare l'obbligo del pagamento degli interessi sul debito ed al rimborso del capitale, con inevitabili riflessi positivi sul consolidamento della posizione assunta in termini di merito creditizio.

Per quanto riguarda le variabili esplicative, al di là di quelle correlate ai risultati di bilancio, che per loro natura sono in qualche misura dipendenti dalle capacità imprenditoriali, ve ne sono altre fortemente caratterizzanti l'agricoltura regionale. Si fa riferimento, in particolare, alle variabili di ordinamento produttivo come l'orticolo ed il floricolo, che sono gli indirizzi produttivi che valorizzano meglio le strutture aziendali presenti nella regione, soprattutto di quelle limitrofe ai grandi centri urbani e di quelle localizzate nelle aree di antica tradizione orticola, caratterizzate dalla presenza di aziende di piccole dimensioni, con operatori con alta qualificazione professionale e da elevati valori fondiari. Va sottolineato, inoltre, come i risultati siano coerenti nel delineare un quadro caratterizzato da un sostanziale effetto negativo esercitato dai miglioramenti fondiari, sia in termini patrimoniali che di bilancio economico. Ciò dimostra come la capacità di rimborso dei prestiti si focalizzi sugli aspetti inerenti strettamente la produzione di liquidità da parte dell'azienda, indipendentemente dalla disponibilità di componenti del capitale fondiario capace di fungere da garanzia.

Nel complesso, quindi, se da un lato la situazione generale dell'agricoltura campana non appare particolarmente preoccupante in termini di rispetto delle obbligazioni assunte nei confronti dei prestatori dei fondi, dall'altro il ruolo negativo esercitato nella definizione dei rating dalle variabili connesse agli investimenti fondiari, potrebbe costituire, a tutta prima, un fattore in grado di ostacolare il processo di aggiustamento strutturale e di crescita dell'agricoltura regionale. Tale risultato, senza dubbio paradossale, tuttavia ad una lettura più attenta, va interpretato nel senso che, ai fini della valutazione della probabilità di insolvenza di un'azienda agricola, ciò che conta per il responsabile della concessione dei prestiti è la capacità degli investimenti di generare una liquidità nel breve periodo superiore a quella assorbita per spese di manuten- 
zione ed ammortamento. In pratica, quindi, il rafforzamento patrimoniale delle aziende agirebbe solo in sede post rating, allorquando viene definita l'entità dell'erogazione creditizia.

Più in particolare, i risultati ottenuti dall'applicazione del modello statistico con variabile dipendente ordinale, il segno negativo associato al predittore "grado di intensità fondiaria" (GIET) si riferisce al complesso dei miglioramenti fondiari, mentre se fosse stato possibile sulla base dei dati disponibili introdurre variabili relative a specifiche tipologie di investimenti fondiari, si sarebbe probabilmente messo in evidenza come le opere che migliorano il fondo con effetti positivi sul beneficio fondiario avrebbero esercitato un impatto positivo sul rating, diversamente da quelle, assimilabili in termini estimativi ai comodi positivi, che, pur influenzando positivamente il valore del capitale fondiario, non hanno effetti significativi sui risultati reddituali, ma solo in termini di accrescimento del valore patrimoniale. D'altra parte, se per aggiustamento strutturale s'intende un assetto dell'agricoltura in grado di remunerare più adeguatamente i fattori della produzione, si comprende come siano proprio gli investimenti fondiari capaci di produrre effetti positivi sul reddito nel breve periodo - come gli impianti irrigui fissi, le sistemazioni delle superfici, ecc. - ad avere un ruolo strategico fondamentale nel processo di crescita del settore.

\section{Riferimenti blibliografici}

Adinolfi F., Capitanio F. (2009). Verso nuove politiche di gestione del rischio. Individuazione dei profili di rischio delle aziende agricole italiane attraverso una analisi dei dati RICA. Economia e Diritto Agroalimentare, XIV, 1.

Agri2000, Osservatorio sull'innovazione nelle imprese agricole, Rapporto 2010.

Akerlof G. (1970). The market for lemons: quality uncertainty and the market mechanism. Quaterly Journal of Economics, 84.

Alberici A. (1975). Analisi dei bilanci e previsione delle insolvenze. Milano: Esedi.

Altman E.I. (1968). Financial Ratios, Discriminant Analysis and the Prediction of Corporate Bankruptcy. The Journal of Finance, XXIII, 4.

Altman E.I. (2000). Predicting financial distress of companies: Revisiting the z-score and zeta® models. Stern School of Business: New York University.

Baltas N.C. (1983). Modelling credit and private investment in Greek agriculture. European Review of Agricultural Econonomics, X, 4.

Barry P.J. (2001). Modern capital management by financial institutions: implications for agricultural lenders. Agricultural Finance Review, LXI, 2.

Bauer L.L., Jordan J.P. (1971). A Statistical Technique for Classifying Loan Applications. Agricultural Experiment Statistical 476. University of Tennessee.

Beaver W.H. (1966). Financial Ratios as Predictors of Failure. Journal of Accounting Reseach, 4.

Beaver W.H. (1968). Market Prices, Financial Ratios and the Prediction of Failure. Journal of Accounting Research, 2. 
Benjamin C., Phimister E. (1997). Transaction costs, farm finance and investment. European Review of Agricultural Economics, XXIV, 3.

Blandford D., Hill B. (2006). Helping European Agriculture to Adjust - A Proactive Approach to Agricultural Policy, EuroChoices, V, 3.

Blum M. (1974). Failing Company Discriminant Analysis. Journal of Accounting Reseach, 1.

Brinegar G.K., Fettig L.P. (1968). Some Measures of the Quality of Agricultural Credit. National Bureau of Economic Research, Tech. Paper 19. Cambridge.

Cameron A.C., Trivedi P.K. (2005). Microeconometrics - Methods and Applications. Cambridge: Cambridge University Press.

Caputo N. (2008). Stato ed evoluzione dei principi di contabilità in agricoltura. In: Crivellaro P., Venceslai G., Grassa R. e Caputo N., Il nuovo credito bancario alle imprese agricole. Bologna: Edagricole.

Collins R.A., Green R.D. (1981). Statistical Methods for Bankruptcy Forecasting. Journal of Economics and Business, XXXIV, 4.

Crivellaro P. (2008). Il nuovo rapporto banche e imprese agricole: dal conflitto alla partership. In: Crivellaro P., Venceslai G., Grassa R. e Caputo N., Il nuovo credito bancario alle imprese agricole. Bologna: Edagricole.

Cupo P., Di Domenico M. (2008). La valutazione del merito creditizio in agricoltura alla luce dell'Accordo Basilea 2: un'applicazione ad un'impresa floricola. Aestimum, 53.

Deakin E.B. (1972). A Discriminant Analysis of Predictors of Business Failure. Journal of Accounting Research, 1.

Dimitras A.I., Zanakis S.H., Zopounidis C. (1996). A survey of business failures with an emphasis on prediction methods and industrial applications. European Journal of Operational Research, XC, 3.

Dixit A., Pyndick R. (1994). Investment under Uncertainty. Princeton: Princeton University Press.

Dunn D.J., Frey T.L. (1976). Discriminant Analysis of Loans for Cash-Grain Farms. Agricultural Finance Review, XXXVI.

Evans C.D. (1971). Analysis of Successful and Unsuccessful Farm Loans in South Dakota. Washington DC: Economic Research Service, USDA.

Fitzpatrick P.J. (1932). A comparison of the ratios of successful industrial enterprises with those of failed companies. The Accountants Publ. Co.

Fontana E. (2010). Valutazione delle aziende agricole per la concessione del credito agrario. Rivista di Economia Agraria, LXI, 1.

Gallagher R.L. (2001). Distinguishing Characteristics of Unsuccessful versus Successful Agribusiness Loans. Agricultural Finance Review, LXI, 1.

Greene W.H. (2000). Econometric Analysis. New Jersey: Prentice Hall Upper Saddle River.

Guarini R., Tassinari F. (1990). Statistica Economica. Problemi e metodi di analisi. Bologna: Il Mulino.

Gustafson C.R., Pederson G.D., Gloy B.A. (2005). Credit Risk Assessment. Agricultural Finance Review, LXV, 2.

Hardy W.E., Adrian J.L. (1985). A Linear Programming Alternative to Discriminant Analysis in Credit Scoring. Agribusiness, I, 4.

Hardy W.E., Weed J.B. (1980). Objective Evaluation for Agricultural Lending. Southern Journal of Agricultural Economics, XII, 1. 
Huttel S., Mubhoff O., Odening M. (2010). Investment reluctance: irreversibility or imperfect capital markets?, European Review of Agricultural Economics, XXXVII, 1.

INEA (2005). Annuario dell'agricoltura italiana. Roma: INEA.

INEA (2006). Annuario dell'agricoltura italiana. Roma: INEA.

INEA (2007). Annuario dell'agricoltura italiana. Roma: INEA.

INEA (2008). Annuario dell'agricoltura italiana. Roma: INEA.

INEA (2009). Annuario dell'agricoltura italiana. Roma: INEA.

ISTAT (2009). Indagine sulla struttura e sulle produzioni delle aziende agricole. ISTAT.

Johnson R.B., Hagen A.R. (1973). Agricultural Loan Evaluation with Discriminant Analysis. Southern Journal of Agricultural Economics, XV, 1.

Lewis P.E.T., Hall N.H., Savage C.R., Kingston A.G. (1988). Taxation, cost of capital and investment in Australian agriculture. Australian Journal of Agricultural Economics, XXXII, 1.

Lucarelli C. (2006). Dal credito agrario per l'agricoltore alla finanza per l'imprenditore agricolo. Agriregionieuropa, 4.

Luerti A. (1992). La previsione dello stato di insolvenza delle imprese. Milano: Etas.

Lufburrow J., Barry P.J., Dixon B.L. (1984). Credit Scoring for Farm Loan Pricing. Agricultural Finance Review, XLIV.

Mauri A. (1990). Considerazioni sul profilo finanziario delle aziende agricole a conduzione familiare. Il Risparmio, XXVIII, 1.

Merwin C.L. (1942). Financing small corporations in five manufacturing industries, 1926-1936. New York: National Bureau of Economic Research.

Miller L.H., LaDue E.L. (1989). Credit Assessment Models for Farm Borrowers: A Logit Analysis. Agricultural Finance Review, XLIX.

Modigliani F., Miller M. (1958). The Cost of Capital, Corporation Finance and The Theory of Investment, American Economic Review, XLVIII, 3.

Mortensen T., Watt D.L., Leistritz L. (1988). Predicting Probability of Loan Default. Agricultural Finance Review, XLVIII.

Ohlson J.A. (1980). Financial Ratios and the Probabilistic Prediction of Bankruptcy. Journal of Accounting Research, XVIII, 1.

Peeters K. (2009). A Competitive, Sustainable and Diverse Agriculture: A View of the CAP Beyond 2013, EuroChoices, IX, 2.

Ravi Kumar P., Ravi V. (2007). Bankruptcy prediction in banks and firms via statistical and intelligent techniques - A review. European Journal of Operational Research, CLXXX, 1.

Roggi O. (2008). Rischio d'impresa, valore e insolvenza. Milano: FrancoAngeli.

Scaramuzzi S. (2002). Il ruolo delle banche locali nello sviluppo rurale: il caso della floricoltura della Valdinievole. In: Basile E. e Romano D., a cura di, Sviluppo rurale: società, territorio, impresa. Milano: FrancoAngeli.

Smith R., Winakor A. (1935). Changes in financial structure of unsuccessful industrial companies. Bureau of Business Research, 51.

Siglitz J., Weiss A. (1981). Credit rationing in markets with imperfect information, American Economic Review, LXXI, 3.

Tarditi S. (1992). Strumenti per l'aggiustamento strutturale dell'agricoltura italiana. In: De Meo G., a cura di, Il governo dell'adattamento dell'agricoltura italiana: istituzioni e strumenti, Atti del XXVIII convegno di studi della SIDEA, Reggio Calabria, 26-28 settembre 1991, Bologna: il Mulino. 
Turvey C.G. (1991). Credit Scoring for Agricultural Loans: A Review with Applications. Agricultural Finance Review, LI.

White H. (1980). A heteroskedasticity-consistent covariance matrix estimator and a direct test for heteroskedasticity. Econometrica, XLVIII, 4.

Winkelmann R., Boes S. (2006) (eds.). Analysis of Microdata. Berlin: Springer.

Vercammen J. (2007). Farm bankruptcy risk as a link between direct payments and agricultural investment, European Review of Agricultural Economics, XXXIV, 4.

Zech L., Pederson G. (2003). Predictors of Farm Performance and Repayment Ability as Factors for Use in Risk-Rating Models. Agricultural Finance Review, LXIII, 1.

Ziari H.A., Leatham D.J., Turvey C.G. (1995). Application of Mathematical Programming in Credit Scoring of Agricultural Loans. Agricultural Finance Review, LV.

\section{Summary}

The factors influencing the credit-worthiness of the farms: an application in Campania

Beyond dealing with the negative consequences of the economic crisis, the globalisation of the agricultural products market and the declining of direct payment by the new course that, most probably, will be undertake by the CAP, more attention should be addressed to competitiveness and entrepreneurship by the regional farmers, especially for those farms whose output are products with a low degree of differentiation. For this reason it is unavoidable to promote a structural adjustment of the farms, in order to generate acceptable returns to the factors of production. The analysis is based on a quantitative evaluation by applying an ordered probit statistical model, by which one has indentified the most significant variables influencing the credit-worthiness of the regional farms. The results show the positive impact of the variables connected with liquidity and the negative impact of the land improvements, with the consequence that could be hampered the structural adjustment and the growth of the regional agriculture.

JEL Codes: $Q 14$

Key words: agricultural credit, credit-worthiness, rating 


\section{Copyright $\odot$ FrancoAngeli}

N.B: Copia ad uso personale. È vietata la riproduzione (totale o parziale) dell'opera con qualsiasi mezzo effettuata e la sua messa a disposizione di terzi, sia in forma gratuita sia a pagamento. 